\title{
Systematic review of preoperative mandibular canal position as it relates to postoperative neurosensory disturbance following the sagittal split ramus osteotomy
}

\author{
J. Rich ${ }^{1}$, B. A. Golden ${ }^{2}$, and C. Phillips ${ }^{1}$ \\ ${ }^{1}$ Department of Orthodontics, School of Dentistry, University of North Carolina, Chapel Hill, North \\ Carolina, USA \\ ${ }^{2}$ Department of Oral and Maxillofacial Surgery, School of Dentistry, University of North Carolina, \\ Chapel Hill, North Carolina, USA
}

\begin{abstract}
The purpose of this study was to review the current literature for the relationship between the preoperative position of the mandibular canal on three-dimensional (3D) radiographic imaging and postoperative neurosensory disturbance (NSD) following a sagittal split ramus osteotomy (SSRO). A literature search was conducted using PubMed, EMBASE, and the Cochrane Database for articles published from 1 January 2000 through 31 December 2013. Studies that included preoperative 3D imaging and assessment of NSD after surgery were reviewed. Study sample characteristics and results were extracted. Of the 69 articles identified, seven met the inclusion and exclusion criteria. There was no standardization for measuring the canal position or for evaluating NSD. General consensus was that the less space between the mandibular canal and the outer border of the buccal cortex the more frequent the occurrence of NSD. Increased bone density also appeared to contribute to a higher incidence of NSD. Utilization of 3D images to locate and measure the position of the mandibular canal is not standardized. Advances in 3D imaging and evaluation tools allow for new methodologies to be developed. Early attempts are informative, but additional studies are needed to verify the relationship between the location of the nerve and NSD following surgery.
\end{abstract}

\section{Keywords \\ mandibular canal; inferior alveolar canal; neurosensory disturbance; sagittal split ramus osteotomy; 3D imaging; CBCT; cone beam computed tomography}

\footnotetext{
(C) 2014 International Association of Oral and Maxillofacial Surgeons. Published by Elsevier Ltd. All rights reserved. Correspondence: C. Phillips, Department of Orthodontics, School of Dentistry, University of North Carolina, CB\#7450, Chapel Hill, NC 27599, USA, Tel: +1 919537 3942, Fax: +1 919843 8864, ceib_phillips@unc.edu.

Competing interests: None declared.

Ethical approval: Not required.

Patient consent: Not required.

Publisher's Disclaimer: This is a PDF file of an unedited manuscript that has been accepted for publication. As a service to our customers we are providing this early version of the manuscript. The manuscript will undergo copyediting, typesetting, and review of the resulting proof before it is published in its final citable form. Please note that during the production process errors may be discovered which could affect the content, and all legal disclaimers that apply to the journal pertain.
} 


\section{Introduction}

Recent reports suggest that up to $40 \%$ of patients who have a sagittal split ramus osteotomy (SSRO) experience persistent (longer than 6 months) neurosensory alteration ${ }^{1-3}$.

Several authors have suggested that the anatomical position of the mandibular canal and surrounding bone quality before surgery may be related to the likelihood of nerve injury during an SSRO ${ }^{1,4-7}$. Others have suggested that the placement of osteotomy cuts and/or fixation placement may be important contributors to the possibility of nerve injury ${ }^{3,8}$. Twodimensional (2D) cephalograms have been used routinely to address maxilla-mandible discrepancies at skeletal maturity and the stability of orthognathic surgery procedures. However, these methods are not sufficiently sensitive or discriminatory to quantify the position of the inferior alveolar canal within the mandible prior to surgery, or the surgical placement of osteotomy cuts or fixation ${ }^{3,9}$.

The use of medical computed tomography (CT) or cone beam computed tomography (CBCT) and three-dimensional (3D) superimposition tools now allow 3D quantification as well as the assessment of surface adaptations using surface distances or shape correspondence not possible with 2D landmark linear or angular measures ${ }^{10}$. The purpose of this study was to review published reports using 3D volumes to assess the position of the mandibular canal preoperatively as it relates to neurosensory disturbances after SSRO surgery.

\section{Methods}

\section{Eligibility criteria}

Longitudinal studies that incorporated 3D imaging obtained before sagittal split osteotomy and post-surgery assessment of neurosensory disturbance (NSD) were investigated. Only articles written between 1 January 2000 and 31 December 2013 and in English were included.

\section{Information sources and search}

Relevant studies were located by searching the National Library of Medicine (PubMed), EMBASE, and the Cochrane Database. The related citations function in PubMed was used to identify additional articles. The search and study selection were carried out independently by two reviewers (JR and CP). The terms used in the search strategy were the following: (1) 'sagittal split', (2) 'neurosensory disturbance', (3) 'mandibular canal', and (4) 'inferior alveolar nerve'. Searches were conducted by filtering studies identified through a combination of search terms 1 and 2 using search terms 3 and 4 separately. Titles and full text abstracts were initially reviewed for eligibility. The full-text of eligible studies was obtained for data extraction (Fig. 1) 


\section{Data collection process}

The following information was extracted from each article: sample characteristics (sample size, gender, age, presenting skeletal disharmony), length of follow-up, measurement(s) obtained from 3D images, assessment of NSD, and results.

\section{Results}

\section{Article selection}

The initial search yielded 69 articles. Thirty-four of these were excluded as duplicate articles. A further 28 were excluded following screening of the titles and full-text abstracts because no 3D imaging (CT) was obtained preoperatively or there was no follow-up assessment of NSD. None of the articles attempted to quantify the postoperative location of the canal, osteotomy cuts, or fixation placement.

\section{Study methods}

While all studies examined the relationship between the position of the inferior alveolar nerve canal (IAN canal) before surgery and NSD following surgery, surgical techniques, CT and NSD measurements, and timing of neurosensory assessment varied. Six studies used spiral CT imaging taken with the occlusal plane perpendicular to the ground and with a slice thickness of $2 \mathrm{~mm}$ to evaluate the location of the IAN canal in relation to the surrounding bone, and one study used CBCT. The location on the mandible selected for measurement of the location of the IAN canal differed. The vast majority of the patients included in these reports were class III. Specifics for each study are outlined below. Table 1 summarizes the 3D measurements and NSD methods.

Yamamoto et al. (2002) ${ }^{4}$ : Twenty patients with a mean age of 22.6 years (range [Au?1] 1640 years) were evaluated. Sixteen of the 20 had a preoperative diagnosis of prognathism. The bone marrow space between the canal and external cortical bone was measured on 2mm-thick CT slices taken from a base plane at the mandibular foramen to $22 \mathrm{~mm}$ below the base plane. The number of consecutive slices showing contact between the canal and cortical bone was recorded as the extent of the contact. The method of Epker was used by the surgeon to determine the bone incision line. Bicortical tandem screws or titanium miniplates were used to provide fixation. NSD was tested bilaterally more than 1 year after surgery using a quantitative algesiometer, a thermocryoesthesiometer, a two-point threshold discriminator, and a small paintbrush for light-touch discrimination. NSD was recorded as present if one or more of the four sensory tests was abnormal when compared to an area of the cheek unaffected by surgery.

Yoshioka et al. (2010) ${ }^{5}$ : Twenty-eight patients with prognathism were assessed; the mean age was 25.8 years (range [Au?1] 18-49 years). Patients were excluded if there was significant mandibular asymmetry, i.e., if the discrepancy for the setback was $>5 \mathrm{~mm}$ from left to right. The distance from the outer buccal cortical margin of the mandible to the buccal aspect of the IAN canal was measured at the mandibular second molar using software connected to the CT scanner. This distance was compared to measurements taken from 30 non-prognathic patients. The outcome of interest was the presence of contact between the 
IAN canal and buccal cortex. The SSRO was performed using the Obwegeser-Dal Pont technique with semi-rigid fixation by titanium miniplate. NSD was measured 3 months after SSRO using a patient questionnaire based on a visual analog scale (VAS) and light touch, brush stroke direction, two-point discrimination, and temperature assessments conducted at six sites.

Yoshioka et al. (2011) 6 : Thirty-five consecutive patients with prognathism were evaluated; the mean age was 25 years (range [Au?1] 18-49 years). All third molars had been taken out at least 1 year previously. A CT scan was taken with bone- tissue windows using a 400 Hounsfield units (HU) window level and a $2000 \mathrm{HU}$ window width. On the cross-sectional $\mathrm{CT}$ at the distal edge of the mandibular second molar, the number of $\mathrm{HU}$ in the bone as an indicator of bone density was measured using the CT scanner software. High HU values are an indicator of higher bone density. The same neurosensory tests as performed by Yoshioka et al. $(2010)^{5}$ were done at 3 months postoperatively to measure NSD.

Yoshioka et al. (2012) 7 : This study included the same 35 consecutive prognathic patients as in Yoshioka et al. (2011) ${ }^{6}$; mean age was 24.6 years (range [Au?1] 18-49 years). The distance from the cortical margin to the $\mathrm{canal}^{5}$ and the HU value ${ }^{6}$ were reported as defined in the previous publications ${ }^{5,6}$. The distance between the superior aspect of the IAN canal and the alveolar crest and the width of the buccal cortical bone distal to the second mandibular molar were also reported. NSD was measured using the same methods as in previous studies done by the author (VAS and neurosensory tests), but the neurosensory assessments were recorded at longer postoperative intervals of 6 and 12 months.

Yamauchi et al. (2012) ${ }^{1}$ : Thirty skeletal class III patients with a mean age of 22 years (range [Au?1] 16-39 years) were studied. All third molars were removed at least 4 months prior to surgery. The bone marrow space between the outer mandibular canal and lateral cortex of the ramus was measured approximately $10 \mathrm{~mm}$ inferior to the occlusal plane on crosssectional CT images. The length between the retromolar and gonion points at the mandibular angle was measured on lateral cephalograms. The retromolar point was defined as the intersection of the tangents to the anterior margin of the mandibular vertical ramus and superior margin of the mandibular body. The Dal Pont surgical technique was used for the SSRO. Care was taken to ensure that the neurovascular bundle was not contained in the proximal segment after the split. Four- or six-hole miniplates and monocortical screws were used to obtain primary stability, followed by intermaxillary fixation (IMF) for approximately 4 days. NSD was assessed bilaterally using discrimination to touch with the sharp head of a mechanical probe at 1,3, and 6 months postoperatively. No criteria specifying the presence/absence of NSD were given.

Kuroyanagi et al. (2013) ${ }^{11}$ : Fifty consecutive patients (48 with prognathism, two with retrognathism) were evaluated; patients were aged 17-44 years. All third molars had been taken out at least 1 year prior, and all patients underwent mandibular osteotomy alone. Distances between the mandibular notch and lingual as well as surgical space medial to the mandibular ramus (space enclosed by the mandible, maxilla, and zygomatic bone) were measured by two independent maxillofacial surgeons on CT images. Clinical sensory tests were done at 1 week, 6 months, and 1 year postoperatively using Semmes-Weinstein 
pressure esthesiometer filaments. NSD was considered positive if the threshold value in one of nine regions was higher than normal (2.83).

Huang et al. (2013) ${ }^{12}$ : One hundred forty-six patients with prognathism, mean age 23.47 years (range [Au?1] 18-39 years), were evaluated and underwent bimaxillary surgery including a bilateral sagittal split osteotomy setback. Preoperative CBCT images were used to measure the distance between the IAN and corresponding buccal cortical bone. The buccal cortical thickness (BCT) was measured as the shortest distance between the inner buccal surface of the mandibular canal and the outer surface of the buccal cortical bone. BCT was measured every $2 \mathrm{~mm}$ from the mandibular foramen to the furcation of the first mandibular molar. NSD was tested at 1 week, 6 weeks, and 1 year using light touch (Semmes-Weinstein monofilament) and pricking pain (dental explorer). NSD was considered positive if there was no response to either light touch or pain.

\section{Major findings}

Yamamoto et al. (2002) $)^{4}$ : At the 1 year or longer postoperative assessment, the prevalence of NSD was $100 \%$ on sides where the canal came into contact with the external cortical plate. No NSD was observed on the 14 sides with a bone marrow space of $1.0 \mathrm{~mm}$ or more. NSD was significantly more likely if the width of the marrow space was $0.8 \mathrm{~mm}$ or less $(P<$ 0.002). Separating the IAN from the external cortical bone without injuring the IAN canal is difficult with a small $(<0.8 \mathrm{~mm})$ or absent bone marrow space.

Yoshioka et al. $(2010)^{5}$ : No significant difference was found in the distance from the buccal aspect of the IAN canal to the outer buccal cortical margin of the mandible between patients with and without mandibular prognathism. The average preoperative distance from the IAN canal to the buccal cortical margin was significantly different for subjects with NSD and without NSD at 3 months post-surgery: the average preoperative mean distance was $4.53 \pm$ $1.03 \mathrm{~mm}$ for subjects with NSD compared to $7.11 \pm 1.10 \mathrm{~mm}$ preoperatively in subjects without NSD at 3 months. The shorter the distance from the buccal aspect of the IAN canal to the outer buccal cortical margin in the mandibular second molar region, the more likely a subject was to present with NSD. At 3 months postoperatively, a higher proportion of women $(42.9 \%)$ had NSD than men $(7.1 \%)(P=0.04)$.

Yoshioka et al. (2011) ${ }^{6}$ : The difference in the preoperative average HU of mandibular bone was significantly different between men and women $(P<0.05)$. Eighteen of the 35 surgery subjects presented with NSD at 3 months postoperatively, with a significantly higher rate of NSD in women $(P=0.04)$. In relation to bone density, the greater the number of $\mathrm{HU}$ in the mandibular bone at the distal edge of the second mandibular molar, the more likely NSD was observed postoperatively $(P<0.001)$. In subjects with unilateral NSD, the number of $\mathrm{HU}$ on the affected side was significantly higher than on the unaffected side $(P<0.01)$ suggesting that high bone density might require more pressure for the osteotomy split and thereby increase the likelihood of damage to the nerve.

Yoshioka et al. (2012) $)^{7}$ : Females had a significantly higher rate of NSD after SSRO at 6 months $(P=0.043)$ and 12 months $(P=0.047)$ than males [Au?2]. The shorter the distance from the buccal aspect of the IAN canal to the outer buccal cortical margin, the more likely 
NSD occurrence was at both 6 months $(P<0.01)$ and 12 months $(P<0.01)$. Resolution of NSD between 3 and 12 months was more likely if the preoperative distance was greater than $6 \mathrm{~mm}$ compared with distances less than $6 \mathrm{~mm}$. Bone quality (measured in HU) impacted healing as well. Subjects with values less than $300 \mathrm{HU}$ were more likely to experience resolution of NSD between 3 months and 12 months $(P<0.0001)$ compared to those with values greater than $300 \mathrm{HU}$.

Even though the samples were the same or overlapped, the three articles by Yoshioka et al. ${ }^{5-7}$ were included because the $\mathrm{CT}$ measurements and timing of assessment differed.

Yamauchi et al. (2012) ${ }^{1}$ : Although no criteria for the presence of NSD were given, the overall occurrence of NSD was reported as $25 \%$ at 1 month, $15 \%$ at 3 months, and $11.7 \%$ at 6 months. A decreased frequency of NSD was correlated with an increased bone marrow space. At 1 month postoperatively, the incidence of NSD in those with a marrow space of 2$3 \mathrm{~mm}$ was $5.9 \%$, significantly less than the $57.1 \%$ of patients experiencing NSD with a preoperative marrow space of $\unlhd \mathrm{mm}(P=0.002)$. Patients with a longer distance from the retromolar to gonion points and a small bone marrow space were significantly more likely to present with NSD at 6 months than those with a shorter distance and larger marrow space ( $P$ $=0.006)$.

Kuroyanagi et al. (2013) ${ }^{11}$ : Lower lip hypoesthesia was seen in $33 \%$ of operated sides at 1 week postoperatively, and $11 \%$ at 6 months. Only $2 \%$ of operated sides showed NSD at 1 year following surgery. The surgical space on the medial side of the mandibular ramus was significantly different between patients with and without NSD at 1 week $(P=0.006)$ and 6 months $(P=0.001)$. A distance of less than $1.5 \mathrm{~mm}$ between the buccal aspect of the mandibular canal and the buccal cortex, and a wide surgical space of $195 \mathrm{~mm}^{2}$ or more on the medial side of the ramus, were associated with a statistically significant higher risk of NSD following surgery $(P<0.001)$.

Huang et al. (2013) ${ }^{12}$ : Numbness of the lower lip was found in $32.5 \%$ of operated sides at 1 week postoperatively. Decreased buccal cortical thickness was identified throughout the length of the mandibular canal in those experiencing NSD compared with those having normal sensation. Thickness of the buccal cortex was an average of $5.67 \pm 0.77 \mathrm{~mm}$ in the NSD group, which was thinner than in the non-NSD group, mean $5.96 \pm 0.75 \mathrm{~mm}$. For both men and women, the buccal cortex was thinner in those who experienced NSD, although the location from the mandibular foramen of the statistically significant differences in thickness differed for men and women.

\section{Discussion}

The overall quality of life improves substantially for the vast majority of patients who have mandibular corrective jaw surgery ${ }^{13-15}$. However, NSD constitutes a notable morbidity for a minority of patients. Virtually all patients who have a mandibular osteotomy experience a neurosensory alteration in the short-term after surgery, and the timeline for resolution likely depends on any number of factors including the type of skeletal deformity as it relates to regional anatomy, osteotomy technique and tools, the amount and direction of surgical 
movement as it relates to nerve position and tension, the amount of required surgical manipulation of the neurovascular bundle intraoperatively, the experience of the operator, the type and location of fixation, patient age, and the time allowed for return of sensation. In the studies reviewed, $2 \%{ }^{11}$ to $17 \%$ of sides operated ${ }^{7}$ were reported as experiencing NSD at 1 year. However, previous publications have discussed the variability in the reporting of the percentage of NSD depending on the method of assessment ${ }^{3}$. Patient self-report findings indicate that more than $60 \%$ of patients report NSD 6 months after surgery ${ }^{16}$ and the alteration can persist for up to 2 years in more than $10 \%$ of the patients ${ }^{17-19}$. Currently, there is no generally accepted, standard method of assessing sensory disturbances in the distribution of the IAN following mandibular osteotomy or on the sites to be assessed, which makes the comparison of findings from published reports difficult ${ }^{3,20}$. A summary of the neurosensory methods employed in the reviewed articles is described in Table 1.

The reports summarized in this review sought to clarify the risk of NSD by focusing on the preoperative IAN canal position ${ }^{1,4-7}$. The position of the canal is particularly relevant to postoperative NSD following SSRO because the canal position impacts and is impacted by osteotomy design and fixation techniques. Novel technologies and software to evaluate the canal are emerging with the availability and utility of modern medical CT and CBCT data. Nevertheless, omitted from any of the studies was the surgeon's operative assessment of the neurovascular bundle during the procedure. Fridrich et al. in 1995 proposed a five-point ordinal rating scale for surgeons to use in the operating room that could be incorporated easily in studies as a possible confounder. The rating scale ranges from grade 1, 'nerve encased within the bony canal and not visualized in the distal segment', to grade 5 , 'nerve transected 21 .

The optimal utilization of 3D innovative techniques continues to be elucidated and is not well standardized. To date, measurements of the IAN canal in relation to the lateral border of the mandible have consistently been done using cross-sections of CT (spiral or cone beam) images. However, within these studies there remains notable variability in the methodology for obtaining these measurements. In some reports the location of the canal was only measured at one location (i.e., the mandibular second molar) ${ }^{5-7}$; while in others it was measured at "whichever slice showed the canal closest to the cortical plate"4.

Additionally, the cross-sectional images that were examined were not standardized to be sliced perpendicular to the ramus, thus creating the potential for a larger measurement than the actual distance.

There is currently insufficient evidence with respect to the measurement on the image that best predicts the likelihood of NSD for routine use in treatment planning or for setting appropriate patient expectations with respect to NSD. Surgical technique may be altered on a case-to-case basis to reflect the surgeon's subjective concern relative to the preoperative location of the canal if a preoperative CT or CBCT is taken.

Another challenge of particular interest for SSRO is that no studies have used 3D imaging to examine the location of the canal after healing has occurred. The unique problem following this technique is that the neurovascular bundle is typically released from the osseous canal during the procedure and assumes a new position between the proximal and distal segments 
along the osteotomy. This complicates the task of identifying the true neurovascular bundle versus the osseous canal. Assessing the effects of fixation and segment adaptation as they relate to the nerve itself and NSD outcomes requires further work to clarify how the anatomy of the subject relates to the image data in this circumstance.

Even so, early attempts to find associations are informative. Presurgery, an increased distance between the canal and cortical bone is associated with a lower incidence of postoperative $\mathrm{NSD}^{1,4,5}$, while greater bone density is associated with a higher incidence of postoperative $\mathrm{NSD}^{6}$. No human data are currently available on the impact of osteotomy or fixation placement on the incidence of NSD. However, from a short post-surgery assessment comparing monocortical and bicortical fixation in a monkey model, there is an indication that IAN function was better after plate fixation than after screw fixation ${ }^{22}$.

With advances in imaging techniques and more robust tools for dealing with 3D images, new methods and standards for evaluating the position of the mandibular canal in relation to the surrounding anatomy are needed. In addition, the current studies almost exclusively enrolled prognathic patients. Since the amount and direction of surgical movement as it relates to nerve position and tension may also affect the occurrence and persistence of NSD, additional studies on patients with other presenting characteristics, retrognathy/asymmetry, as well as studies with long-term ( $\geq 12$ months) follow-up are needed to more accurately correlate the preoperative and postoperative position of the IAN canal with persistent postoperative NSD.

\section{Acknowledgments}

Funding: Research reported in this publication was supported by NIDCR of the National Institutes of Health under award number R01DE005215. The content is solely the responsibility of the authors and does not necessarily represent the official views of the National Institutes of Health.

\section{References}

1. Yamauchi K, Takahashi T, Kaneuji T, Nogami S, Yamamoto N, Miyamoto I, Yamashita Y. Risk factors for neurosensory disturbance after bilateral sagittal split osteotomy based on position of mandibular canal and morphology of mandibular angle. J Oral Maxillofac Surg. 2012; 70:401-406. [PubMed: 21549489]

2. Colella G, Cannavale R, Vicidomini A, Lanza A. Neurosensory disturbance of the inferior alveolar nerve after bilateral sagittal split osteotomy: a systematic review. J Oral Maxillofac Surg. 2007; 65:1707-1715. [PubMed: 17719387]

3. Phillips C, Essick G. Inferior alveolar nerve injury following orthognathic surgery: a review of assessment issues. J Oral Rehabil. 2011; 38:547-554. [PubMed: 21058973]

4. Yamamoto R, Nakamura A, Ohno K, Michi KI. Relationship of the mandibular canal to the lateral cortex of the mandibular ramus as a factor in the development of neurosensory disturbance after bilateral sagittal split osteotomy. J Oral Maxillofac Surg. 2002; 60:490-495. [PubMed: 11988921]

5. Yoshioka I, Tanaka T, Khanal A, Habu M, Kito S, Kodama M, Oda M, Wakasugi-Sato N, Matsumoto-Takeda S, Fukai Y, Tokitsu T, Tomikawa M, Seta Y, Tominaga K, Morimoto Y. Relationship between inferior alveolar nerve canal position at mandibular second molar in patients with prognathism and possible occurrence of neurosensory disturbance after sagittal split ramus osteotomy. J Oral Maxillofac Surg. 2010; 68:3022-3027. [PubMed: 20739116]

6. Yoshioka I, Tanaka T, Khanal A, Habu M, Kito S, Kodama M, Oda M, Wakasugi-Sato N, Matsumoto-Takeda S, Seta Y, Tominaga K, Sakoda S, Morimoto Y. Correlation of mandibular 
bone quality with neurosensory disturbance after sagittal split ramus osteotomy. Br J Oral Maxillofac Surg. 2011; 49:552-556. [PubMed: 21071118]

7. Yoshioka I, Tanaka T, Habu M, Oda M, Kodama M, Kito S, Seta Y, Tominaga K, Sakoda S, Morimoto Y. Effect of bone quality and position of the inferior alveolar nerve canal in continuous, long-term, neurosensory disturbance after sagittal split ramus osteotomy. J Craniomaxillofac Surg. 2012; 40:e178-e183. [PubMed: 22000578]

8. Macintosh RB. Experience with the sagittal osteotomy of the mandibular ramus: a 13-year review. J Maxillofac Surg. 1981; 9:151-165. [PubMed: 6974211]

9. Ludlow JB, Gubler M, Cevidanes L, Mol A. Precision of cephalometric landmark identification: cone-beam computed tomography vs conventional cephalometric views. Am J Orthod Dentofacial Orthop. 2009; 136:312.e1, 312.e10. discussion 312- 313. [PubMed: 19732656]

10. Cevidanes LH, Alhadidi A, Paniagua B, Styner M, Ludlow J, Mol A, Turvey T, Proffit WR, Rossouw PE. Three-dimensional quantification of mandibular asymmetry through cone-beam computerized tomography. Oral Surg Oral Med Oral Pathol Oral Radiol Endod. 2011; 111:757770. [PubMed: 21497527]

11. Kuroyanagi N, Miyachi H, Ochiai S, Kamiya N, Kanazawa T, Nagao T, Shimozato K. Prediction of neurosensory alterations after sagittal split ramus osteotomy. Int J Oral Maxillofac Surg. 2013; 42:814-822. [PubMed: 23265759]

12. Huang CS, Syu JJ, Ko EW, Chen YR. Quantitative evaluation of cortical bone thickness in mandibular prognathic patients with neurosensory disturbance after bilateral sagittal split osteotomy. J Oral Maxillofac Surg. 2013; 71:2153.e1-2153.e10. [PubMed: 24135253]

13. Rustemeyer J, Gregersen J. Quality of life in orthognathic surgery patients: post-surgical improvements in aesthetics and self-confidence. J Craniomaxillofac Surg. 2012; 40:400-404. [PubMed: 21865051]

14. Lee S, McGrath C, Samman N. Impact of orthognathic surgery on quality of life. J Oral Maxillofac Surg. 2008; 66:1194-1199. [PubMed: 18486784]

15. Murphy C, Kearns G, Sleeman D, Cronin M, Allen PF. The clinical relevance of orthognathic surgery on quality of life. Int J Oral Maxillofac Surg. 2011; 40:926-930. [PubMed: 21616638]

16. Phillips C, Essick G, Zuniga J, Tucker M, Blakey GH III. Qualitative descriptors used by patients following orthognathic surgery to portray altered sensation. J Oral Maxillofac Surg. 2006; 64:1751-1760. [PubMed: 17113441]

17. Essick GK, Phillips C, Kim SH, Zuniga J. Sensory retraining following orthognathic surgery: effect on threshold measures of sensory function. J Oral Rehabil. 2009; 36:415-426. [PubMed: 19422435]

18. Phillips C, Kim S, Essick G, Tucker M, Turvey TA. Sensory retraining following orthognathic surgery: effect on patient report of the presence of altered sensation. Am J Orthod Dentofacial Orthop. 2009; 136:788-794. [PubMed: 19962601]

19. Calabria F, Sellek L, Gugole F, Trevisiol L, Bertolasi L, D'Agostino A. The use of sensory action potential to evaluate inferior alveolar nerve damage after orthognathic surgery. J Craniofac Surg. 2013; 24:514-517. [PubMed: 23524729]

20. Poort LJ, van Neck JW, van der Wal KG. Sensory testing of inferior alveolar nerve injuries: a review of methods used in prospective studies. J Oral Maxillofac Surg. 2009; 67:292-300. [PubMed: 19138602]

21. Fridrich KL, Holton TJ, Pansegrau KJ, Buckley MJ. Neurosensory recovery following the mandibular bilateral sagittal split osteotomy. J Oral Maxillofac Surg. 1995; 53:1300-1306. [PubMed: 7562195]

22. Fujioka M, Hirano A, Fuji T. Comparative study of inferior alveolar disturbance restoration after sagittal split osteotomy by means of bicortical versus monocortical osteosynthesis. Plast Reconstr Surg. 1998; 102:37-41. [PubMed: 9655405] 


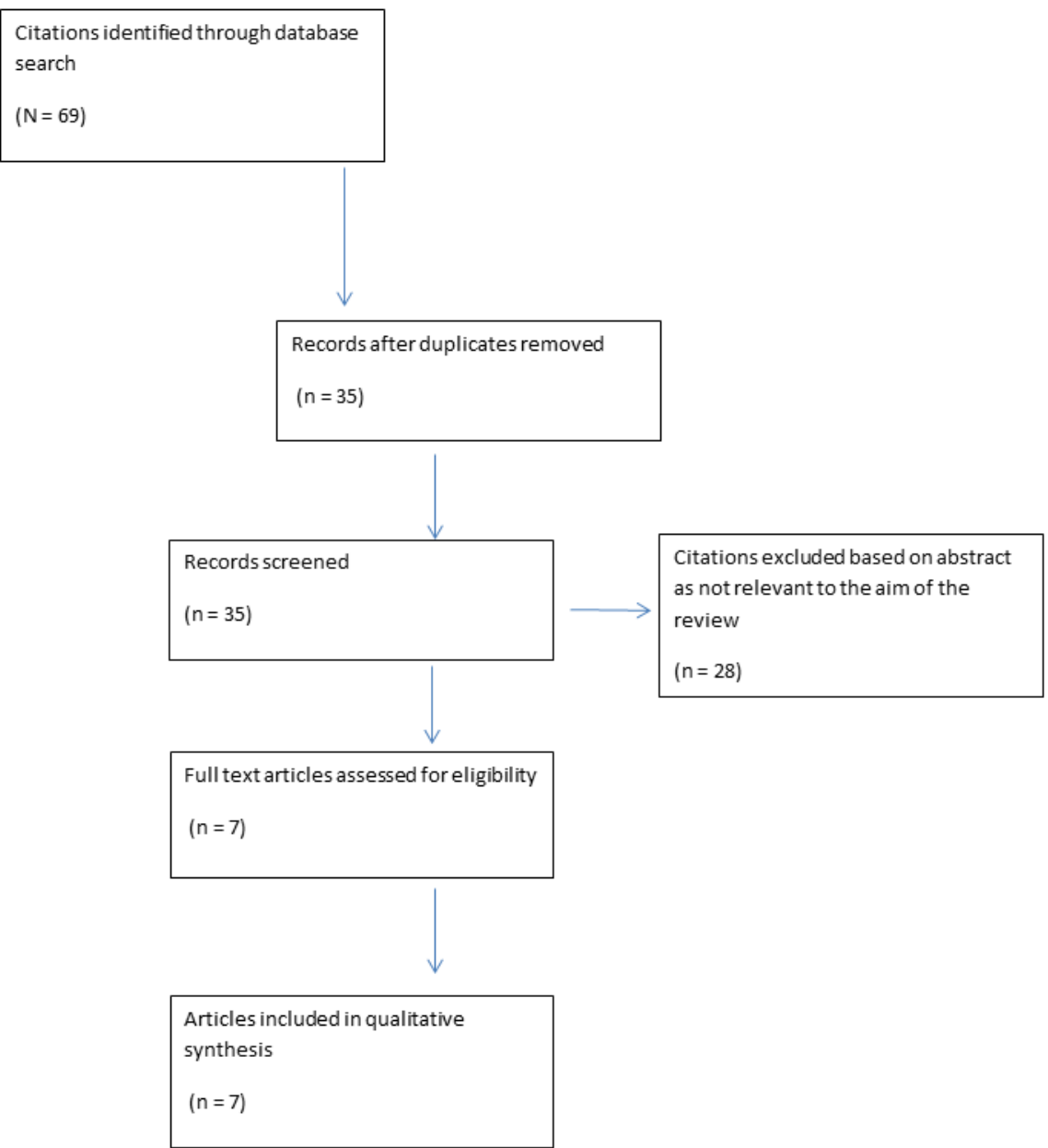

Fig. 1.

Flow diagram summarizing the literature search. 
Table 1

Techniques used for canal measurement and testing for neurosensory disturbance.

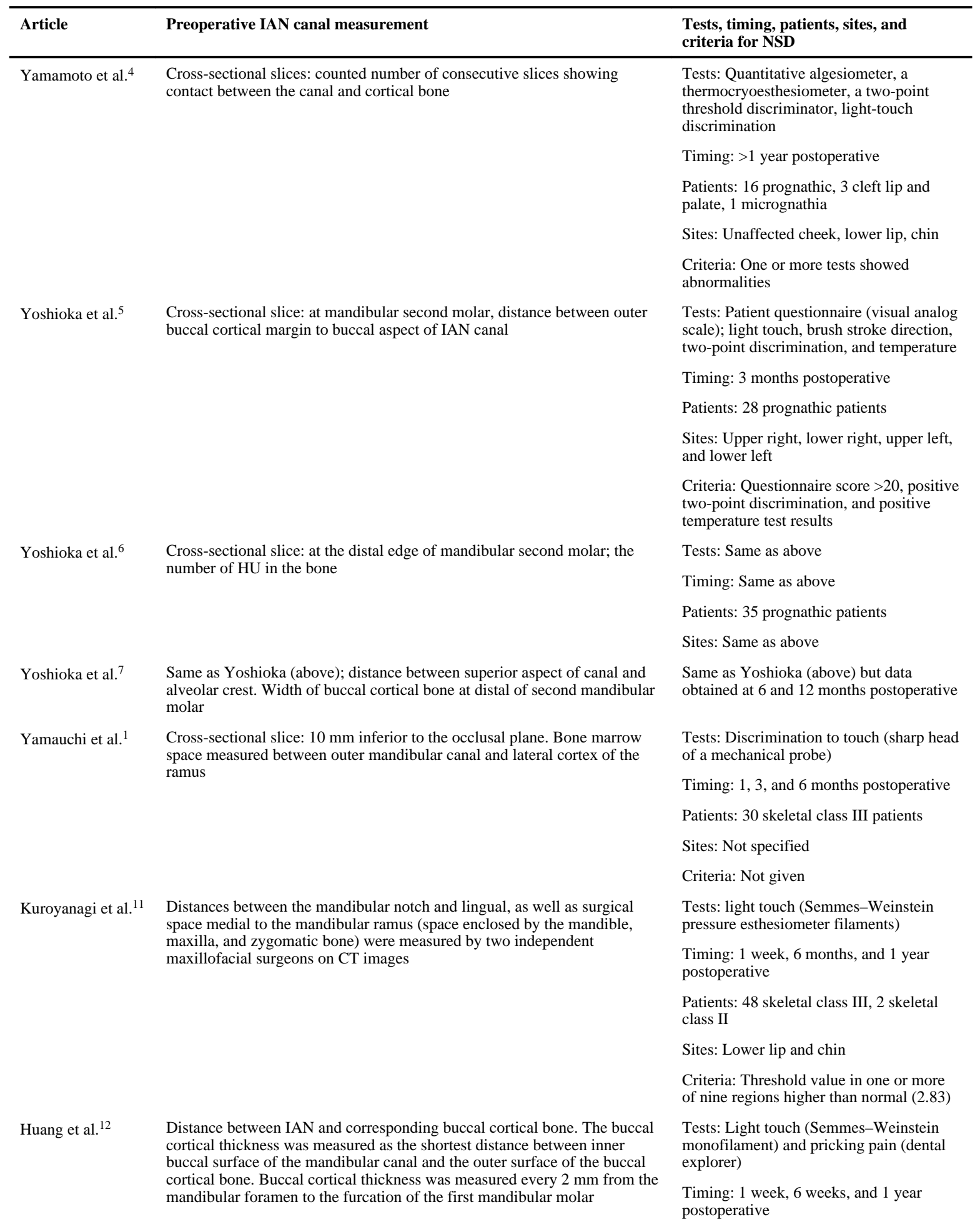




\begin{tabular}{lll}
\hline Article & Preoperative IAN canal measurement & $\begin{array}{l}\text { Tests, timing, patients, sites, and } \\
\text { criteria for NSD }\end{array}$ \\
\hline & Patients: 146 prognathic patients \\
Sites: Lower lip and chin - four zones \\
Criteria: No response to either light touch \\
or pain
\end{tabular}

IAN, inferior alveolar nerve; NSD, neurosensory disturbance; HU, Hounsfield units; CT, computed tomography. 